\title{
Correction to: Transparent nanocellulose-pigment composite films
}

\author{
Camila Honorato ${ }^{1}$, Vinay Kumar ${ }^{2, *}$ (D), Jun $\mathrm{Liu}^{3}$, Hanna Koivula ${ }^{4}$, Chunlin $\mathrm{Xu}^{5,6}$, \\ and Martti Toivakka ${ }^{7}$ \\ ${ }^{1}$ Nanomaterials and Nanotechnologies, Luxembourg Institute of Science and Technology, Maison De L'innovation, 5, Avenue Des \\ Hauts-Fourneaux, 4362 Esch-sur-Alzette, Luxembourg \\ ${ }^{2}$ Biomass Processing and Products, VTT Technical Research Centre of Finland, Tietotie 4 E, 02044 VTT Espoo, Finland \\ ${ }^{3}$ School of the Environment and Safety Engineering, Jiangsu University, 301 Xuefu Road, Zhenjiang 212013, Jiangsu Province, \\ China \\ ${ }^{4}$ Department of Food and Environmental Sciences, University of Helsinki, PL 66, Agnes Sjöberginkatu 2, 00014 Helsinki, Finland \\ ${ }^{5}$ Laboratory of Wood and Paper Chemistry, Åbo Akademi University, Porthansgatan 3, 20500 Åbo/Turku, Finland \\ ${ }^{6}$ Wallenberg Wood Science Center, KTH, The Royal Institute of Technology, 10044 Stockholm, Sweden \\ ${ }^{7}$ Laboratory of Paper Coating and Converting, Centre for Functional Materials, Åbo Akademi University, Porthaninkatu 3, \\ 20500 Turku, Finland
}

\section{Published online:}

18 September 2019

(C) Springer Science+Business

Media, LLC, part of Springer

Nature 2019

Correction to: J Mater Sci (2015) 50:7343-7352 https://doi.org/10.1007/s10853-015-9291-7
Publisher's Note Springer Nature remains neutral with regard to jurisdictional claims in published maps and institutional affiliations.

In the original version of this article, one of the concentrations of CNF suspensions has been reported to be $0.01 \%$ under the results and discussion of rheology measurements. It should have been $0.1 \%$ instead.

The original article can be found online at https:/ / doi.org/10.1007/s10853-015-9291-7.

Address correspondence to E-mail: vinay.kumar@vtt.fi 